\title{
Imaging and Surgical Findings in Patients with Hemi-Laryngopharyngeal Spasm and the Potential Role of MRI in the Diagnostic Work-Up
}

\author{
(D). Avecillas-Chasin, (D) M.G. Kozoriz, (D).R. Shewchuk, (DM.K.S. Heran, and (D) C.R. Honey
}

\begin{abstract}
BACKGROUND AND PURPOSE: Hemi-laryngopharyngeal spasm is a recently discovered condition characterized by episodic coughing and unilateral throat contractions that may lead to severe stridor. These symptoms are caused by a vascular compression of the ipsilateral vagus nerve, typically the PICA. Microvascular decompression of the vagus nerve has been demonstrated to be a potential cure for this neurovascular compression syndrome. The main aim of this study was to clarify the role of MR imaging in the diagnostic work-up of this rare condition.
\end{abstract}

MATERIALS AND METHODS: We describe the imaging and surgical findings of 3 patients from our prospective case series of patients with hemi-laryngopharyngeal spasm from 2015 to 2017. Second, the imaging data of 100 patients (control cohort) with symptoms unrelated to hemi-laryngopharyngeal spasm were reviewed to investigate the rate and degree of neurovascular conflict of the vagus nerve.

RESULTS: All patients with hemi-laryngopharyngeal spasm reported to date have had vascular compression of the vagus nerve due to the PICA. In the control cohort, there was a good interrater agreement in scoring the "contact" and "compression" of the vagus nerve $(\kappa=0.73$. $P=<.001)$. The frequency of contact or compression of the vagus nerve was approximately $50 \%$. The PICA was the most frequent vessel involved in $74 \%$.

CONCLUSIONS: The presence of unilateral neurovascular contact or compression of the vagus nerve does not confirm the diagnosis of hemi-laryngopharyngeal spasm. The MR imaging finding of ipsilateral vascular compression of the vagus nerve is a necessary but not sufficient finding for the diagnosis of hemi-laryngopharyngeal spasm.

ABBREVIATIONS: HELPS = hemi-laryngopharyngeal spasm; MVD = microvascular decompression

$\mathrm{H}$ emi-laryngopharyngeal spasm (HELPS) is a recently discovered condition characterized by episodic coughing and unilateral throat contractions that may lead to severe stridor. These symptoms are caused by a vascular compression of the ipsilateral vagus nerve, typically by the tonsillomedullary segment of the PICA. Similar to hemifacial spasm, the motor component of HELPS (ipsilateral pharyngeal or laryngeal contractions) does not respond to medications but can be ameliorated with ipsilateral botulinum toxin. Microvascular decompression (MVD) of the vagus nerve has been demonstrated to be a potential cure for this neurovascular compression syndrome. ${ }^{1,2}$ The diagnosis of the

Received May 18, 2018; accepted after revision September 4.

From the Department of Surgery (J.A.-C., C.R.H.), Division of Neurosurgery, and Department of Radiology (M.G.K., J.R.S., M.K.S.H.), University of British Columbia, Vancouver, British Columbia, Canada; Vancouver General Hospital (C.R.H.), Vancouver, British Columbia, Canada.

Please address correspondence to Christopher R. Honey, MD, PhD, FRCSC, FACS, Vancouver General Hospital, 8101-2775 Laurel St, Vancouver, BC, V5Z 1M9;

e-mail: chris.honey@telus.net

-- Indicates open access to non-subscribers at www.ajnr.org

http://dx.doi.org/10.3174/ajnr.A5851 more common neurovascular compression syndromes, such as trigeminal neuralgia, glossopharyngeal neuralgia, and hemifacial spasm, often relies on MR imaging of the brain with a 3D balanced steady-state gradient-echo sequence to demonstrate the cranial nerve anatomy and the offending compressing vessel. Many studies have reported a surprisingly high incidence of neurovascular compression of the trigeminal and facial nerves in asymptomatic patients. ${ }^{3-5}$ There is currently no information about the incidence of vascular compression of the vagus nerve, to our knowledge. As HELPS begins to be more widely recognized, we sought to clarify the role of MR imaging in its diagnosis. The aims of this study were the following: 1) to correlate the imaging and surgical findings of patients with HELPS, 2) to estimate the incidence of neurovascular conflict of the vagus nerve in the general population, and 3) to clarify the role of MR imaging in the diagnostic work-up of this rare condition.

\section{MATERIALS AND METHODS}

The clinical research ethics board of the University of British Columbia approved this study (H17-03466). First, we describe the 

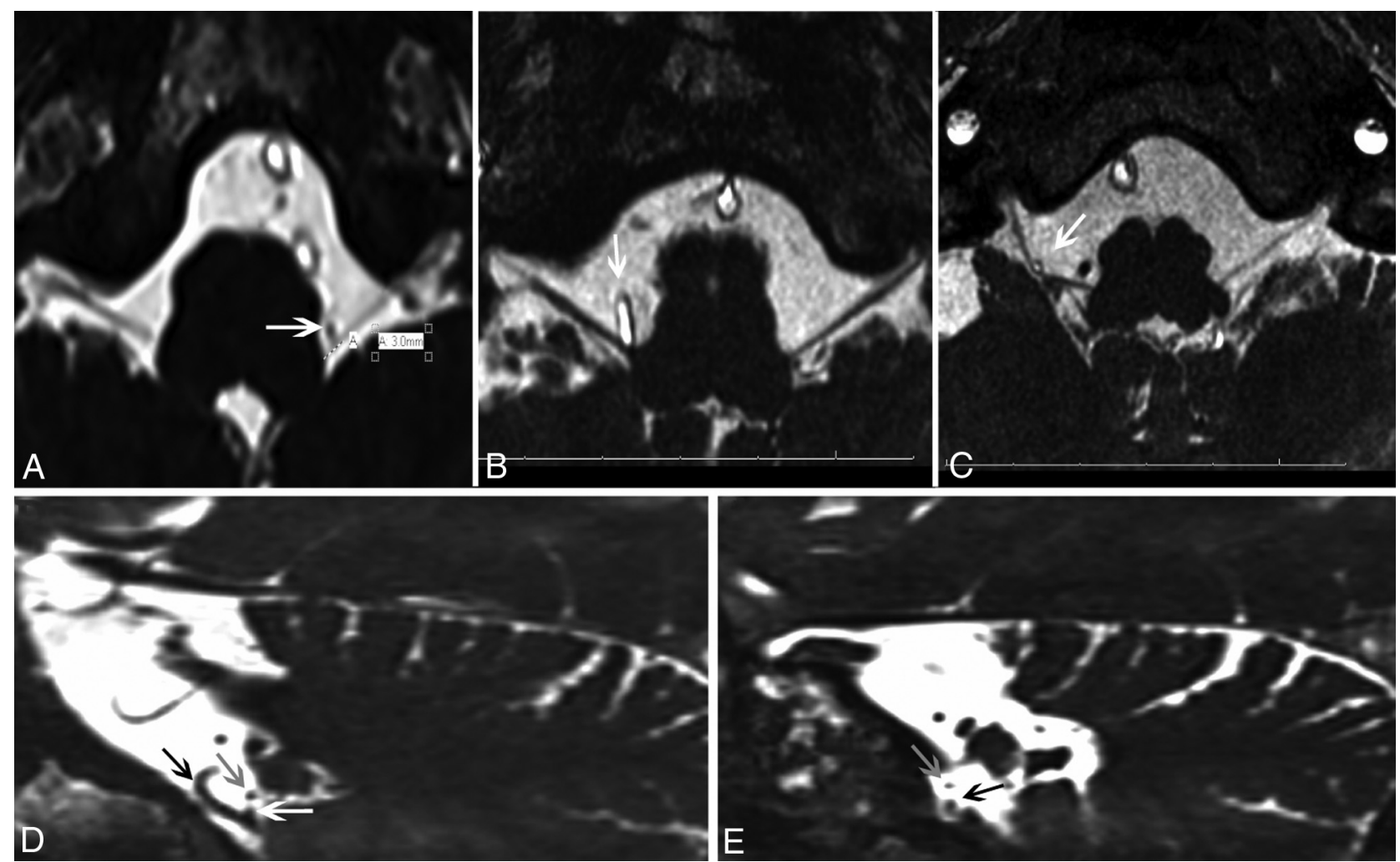

FIG 1. Grading system. The vagus nerve was rated separately for the proximal ( $3 \mathrm{~mm}$ from the brain stem) and the distal portions. A, Grade I: "no vessel contact" (white arrow). B, Grade II: "contact," which was defined as no visible CSF between the blood vessel and the nerve but no displacement of the normal trajectory of the nerve (white arrow). C, Grade III: "compression," which was defined as displacement of the normal trajectory of the nerve (white arrow). D, Grade I: Oblique projection showing the vagus nerve (white arrow) in contact with the PICA (black arrow). The glossopharyngeal nerve (gray arrow) is shown above the vagus nerve. E, Oblique projection of the IX/X complex close to the jugular foramen. It was possible to see the upper glossopharyngeal nerve more anteriorly and superiorly moving lateral into the foramen, and the vagus nerve branches were below this.

imaging and surgical findings of 3 patients from our prospective case series of patients with HELPS from 2015 to 2017. The full clinical description of 3 of these patients has been recently published. ${ }^{2}$ These patients were effectively treated with unilateral MVD of the vagus nerve. Second, the imaging data of 100 patients (control cohort) with symptoms unrelated to HELPS were reviewed to investigate the rate and degree of neurovascular conflict of the vagus nerve. These patients ( 48 men and 52 women) ranged from 24 to 91 years of age and had undergone 1.5T MR imaging of the cerebellopontine angle cisterns with a 3D balanced steadystate gradient-echo sequence (or FIESTA) during 2017 for the following symptoms: tinnitus, headache, isolated facial contractions not consistent with hemifacial spasm, and facial pain not consistent with trigeminal or glossopharyngeal neuralgia. The inclusion criteria for this control cohort of 100 consecutive patients were those with a 3D-CISS or FIESTA sequence on MR imaging with clear visualization of the cerebellomedullary region, no evidence of tumor or vascular malformations, no history of head trauma or surgical procedures in the posterior fossa, and no signs on the FLAIR sequence of multiple sclerosis or other inflammatory or demyelinating conditions.

\section{Neurovascular Conflict Assessment}

Posterior fossa images were acquired on a $1.5 \mathrm{~T}$ platform using a $3 \mathrm{D}$ balanced steady-state gradient-echo sequence. The voxel size was $0.4 \mathrm{~mm}$, the slice thickness was $0.5 \mathrm{~mm}$ with no gap or overlap, and slice interpolation was used in the $\mathrm{z}$-axis. TE and TR were approximately 2.73 and $5.94 \mathrm{~ms}$, respectively. Two researchers (M.G.K., J.A.-C.) evaluated and rated 200 vagus nerves looking for the presence and degree of neurovascular conflict. The grading scale was as follows: grade 1, "no vessel contact"; grade 2, "contact," which was defined as no visible CSF between the blood vessel and the nerve but no displacement of the normal trajectory of the nerve; and grade 3, "compression," which was defined as displacement of the normal trajectory of the nerve (Fig $1 A-D) .{ }^{6,7}$ The rating was performed separately for the transitional zone of the vagus nerve (within $3 \mathrm{~mm}$ from the brain stem) and the cisternal portion of the nerve (henceforth referred to as the proximal and distal portions of the nerve, respectively). ${ }^{8,9}$ The vagus nerve was evaluated along its entire course from the brain stem to the jugular foramen in oblique, axial, and coronal planes. The glossopharyngeal nerve could be identified as the most anterior and superior nerve in the jugular foramen, with the vagus nerve inferior (or caudal) to it (Fig 1E). In a small number of patients $(\sim 5 \%)$, it was not possible to visualize these nerves as separate entities. When this issue occurred, they were evaluated as an IX/X complex. In patients with "contact" or "compression," we recorded the offending vessel and its direction of compression against the vagus nerve. We also simultaneously graded the neurovascular conflict of the trigeminal nerve to determine its rate of 
Clinical and demographic data of our patients with HELPS

\begin{tabular}{|c|c|c|c|c|}
\hline $\begin{array}{l}\text { Patient } \\
\text { No. }\end{array}$ & Age/Sex & Symptoms & $\begin{array}{l}\text { Offending } \\
\text { Vessel }\end{array}$ & $\begin{array}{l}\text { Grading } \\
\text { of NVC }\end{array}$ \\
\hline 1 & $65 / M$ & Episodic throat contractions and coughing & Left PICA & 2 \\
\hline 2 & $43 / \mathrm{F}$ & $\begin{array}{l}\text { Episodic throat contractions, coughing, and vocal } \\
\text { changes }\end{array}$ & Right PICA & 3 \\
\hline 3 & $32 / F$ & $\begin{array}{l}\text { Episodic left-sided throat contractions, choking, } \\
\text { and vocal changes }\end{array}$ & Left PICA & 2 \\
\hline
\end{tabular}

Note:-NVC indicates neurovascular conflict.

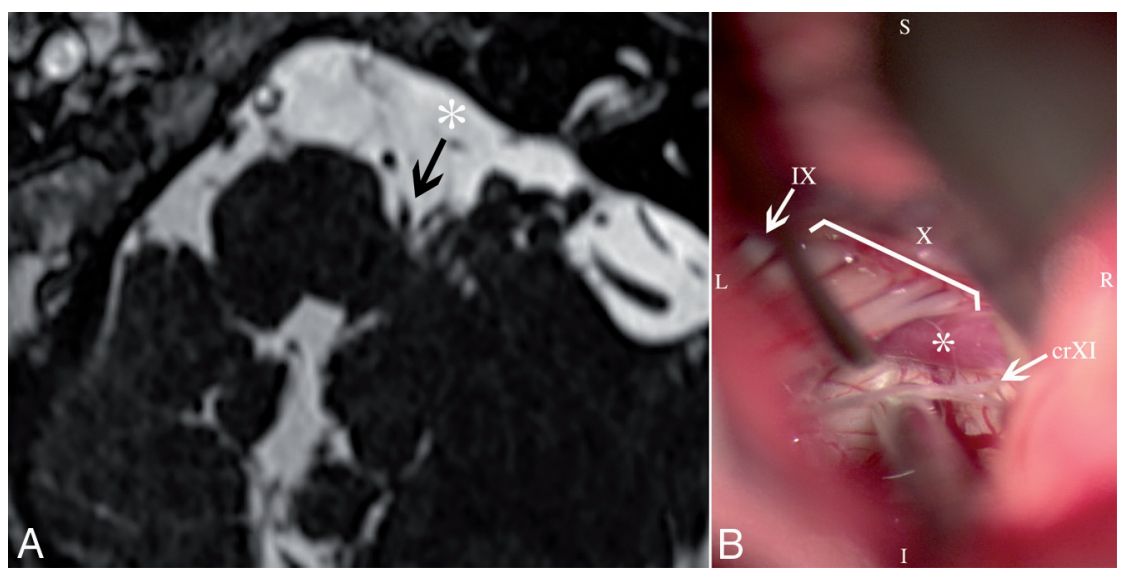

FIG 2. Patient 1. A, MR imaging shows neurovascular conflict of the PICA (arrow) with the proximal part of the vagus nerve. $B$, Intraoperative findings show the loop of the PICA (asterisk) pulsating against the caudal rootlets of the vagus nerve (X). IX indicates glossopharyngeal nerve; crXI, cranial root of the accessory nerve; S, superior; I, inferior; R, right; L, left.

contact/compression as a control to compare our cohort with the other patient groups previously reported in the literature.

\section{Statistical Analysis}

The frequencies for each grade of compression, the offending vessel, and the direction of compression were calculated. The Cohen $\kappa$ coefficient was calculated to determine the level of interrater agreement. A $\kappa$ value $<0.4$ was considered poor agreement, between 0.40 and 0.59 was a fair agreement, between 0.60 and 0.74 was a good agreement, and $\geq 0.75$ was excellent agreement. We used the SPSS software package 22.0 (IBM, Armonk, New York), and a $P$ value $<.05$ was considered significant.

\section{RESULTS}

All patients with HELPS reported to date have had vascular compression of the vagus nerve due to the PICA. The clinical and demographic data of 3 patients are presented in the Table, and their surgical and imaging findings are demonstrated in Figs 2-4. In the control cohort, there was good interrater agreement in scoring the contact and compression of the vagus nerve $(\kappa=0.73$, $P=<.001)$. The proximal portion of the left vagus nerve had $10 \%$ contact and $11 \%$ compression, and the right side had $10 \%$ contact and $1 \%$ compression. The distal portion of the left vagus nerve had $16 \%$ contact and 16\% compression, and the right side had 20\% contact and 15\% compression. The total frequency of contact or compression for the entire left or right vagus nerve was therefore approximately $50 \%$. The vessel in contact or compressing the vagus nerve was the PICA in $74 \%$, the AICA in $14 \%$, the vertebral artery in $6 \%$, and a vein in $6 \%$. The direction of the contact/compression relative to the nerve was from anterior in
$52 \%$, posterior in $24 \%$, inferior in $13 \%$, and superior in $11 \%$. There was excellent interrater agreement in scoring the neurovascular conflict of the trigeminal nerve $(\kappa=0.83, P=<.001)$. There was $54 \%$ (grade 2, 44\%; grade 3, $10 \%$ ) contact or compression of the left trigeminal nerve and 52\% (grade 2, 42\%; grade 3, $10 \%)$ of the right trigeminal nerve.

\section{DISCUSSION}

Patients with hemi-laryngopharyngeal spasm have unilateral neurovascular compression of the vagus nerve. In all our patients, the offending vessel was the PICA and patients were cured following MVD. The 3 examples presented in this study showed neurovascular conflict caused by a PICA loop impacting the vagus nerve from inferior and anterior. Two patients had vascular contact with the proximal portion of the vagus nerve, and 1 had contact with the distal portion of the nerve. According to the grading system used in this study, 2 patients presented with contact, and 1 patient, with compression of the vagus nerve. In this study, we also report that asymptomatic or incidental vascular contact or compression of the vagus nerve was common in our control cohort $(\sim 50 \%)$. We also noted that identification of neurovascular conflict of the vagus nerve is more difficult than for the trigeminal or facial nerve because the vagus nerve rootlets can be in close relationship with the cerebellar tonsil for a variable length of its trajectory. The degree of contrast between the vagus nerve rootlets and the tonsil is much less than between the trigeminal nerve and the CSF.

Three of the first 4 patients successfully treated for HELPS had unilateral compression of the vagus nerve primarily involving the more caudal rootlets. ${ }^{1,2}$ Compression of the rostral rootlets is likely possible, but because of the close proximity of the glossopharyngeal nerve, the resulting clinical presentation is likely to be a combination of painful glossopharyngeal neuralgia and HELPS. We have treated 1 such patient (Prof Christopher Honey Presentation: ESSFN 2018 Oral presentation, September 27, 2018, unpublished results) who had unilateral vagus and glossopharyngeal nerve compression from anterorostrally.

We dichotomized the location of the vagus nerve contact or compression into proximal and distal at $3 \mathrm{~mm}$ from the brain stem. Guclu et $\mathrm{al}^{9}$ reported that the transitional zone of the vagus nerve can vary between 0.45 and $4.2 \mathrm{~mm}$. Our experience with trigeminal neuralgia led us to believe that the vagus nerve could be pathologically affected by a vessel anywhere along its course, but the degree of contact/compression required may be much less where the nerve is tethered proximally. The number of affected patients is too small to comment on this feature, but the data will be available for future analysis. The vagus nerve emerges at the retro-olivary groove in the medulla as several rootlets that ulti- 


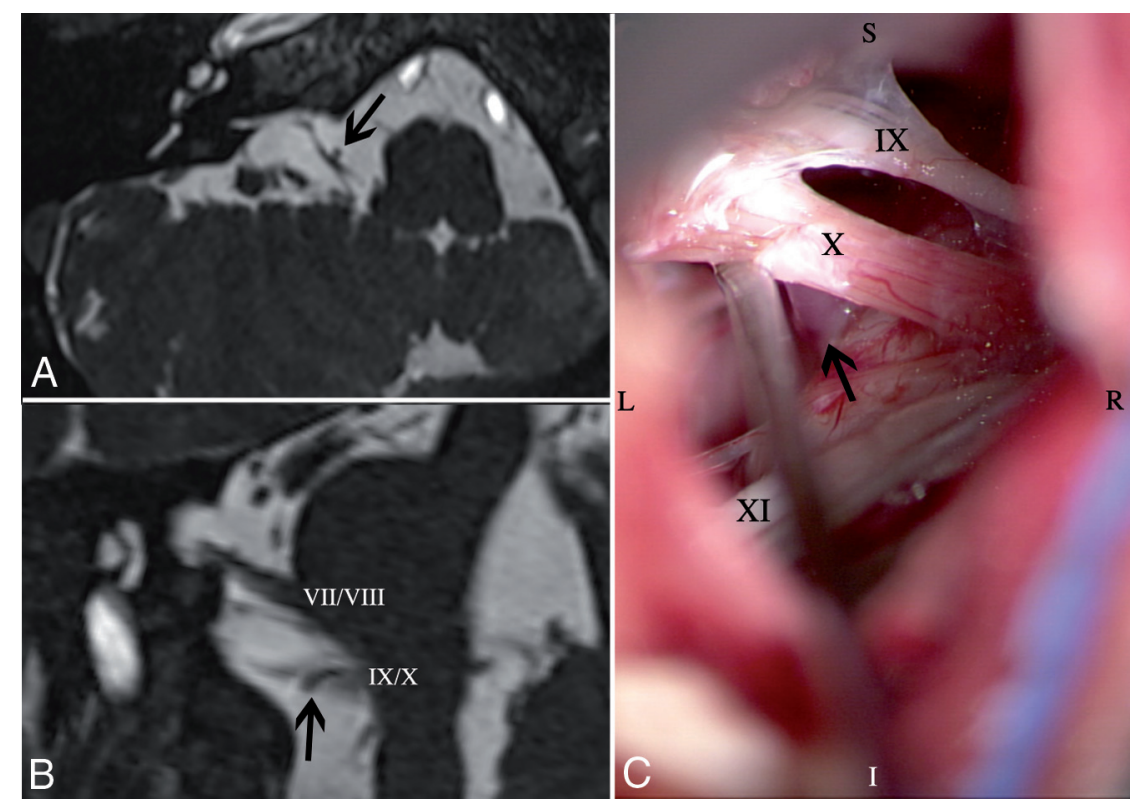

FIG 3. Patient 2. A, Axial view of MR imaging shows compression of the vagus nerve by the right PICA from the anterior direction (arrow). $B$, The coronal-oblique plane shows the vessel loop of the PICA (arrow). VII/VIII indicates the facial and vestibulocochlear nerve complex; IX/X, the glossopharyngeal and vagus nerve complex. $C$, Intraoperative findings confirm that the vessel loop of the PICA (arrow) is pulsating against the anterior aspect of the vagus nerve (X). IX indicates the glossopharyngeal nerve; XI, the accessory nerve; S, superior; I, inferior; R, right; L, left.

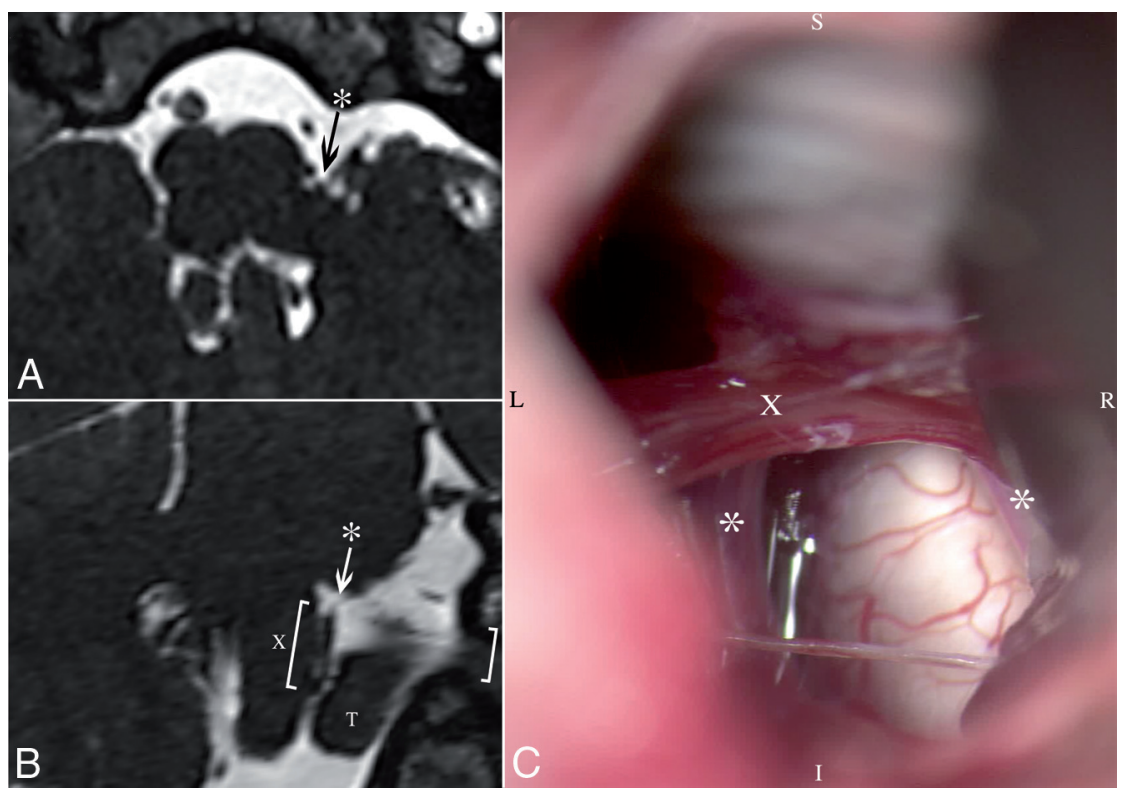

FIG 4. Case 3. A, Axial view of MR imaging shows neurovascular conflict of the left PICA (asterisk) and the vagus nerve. $B$, The coronal-oblique plane shows the loop of the left PICA (asterisk) in contact with the proximal part of the rootlets of the vagus nerve $(X)$, which is also in contact with the left cerebellar tonsil (T). C, Intraoperative findings. After gentle retraction of the cerebellum and part of the left tonsil, the left PICA loop (asterisk) is found in contact with the proximal part of the vagus nerve $(\mathrm{X})$. S indicates superior; I, inferior; R, right; L, left.

mately enter the jugular foramen after a cisternal trajectory. The proximal portion of the nerve is in close proximity with the tonsil and is therefore poorly visualized with standard MR imaging. ${ }^{10}$ 3T MR imaging may be able to delineate the vagus nerve better because of its high signal-to-noise ratio. ${ }^{11}$ Another challenge with the current MR imaging protocols for detecting neurovascular compression is their lack of dynamic information. A vessel loop with its convex apex contacting a nerve will pulsate against it with every heartbeat, while another with its apex directed away from the nerve will not. Both would be reported as "contact" in this and other studies. It remains to be seen whether new dynamic or cine MR imaging sequences will clarify neurovascular compression as they have clarified the dynamics of spinal cord syrinx. There will always be a patient-specific nerve vulnerability to vascular compression that may partially explain why few patients develop symptoms despite the high incidence of neurovascular compression. Our control cohort had approximately $50 \%$ of patients with contact or compression of the trigeminal nerve. This was within the range of the previously reported studies by Antonini et al $(17 \%),{ }^{12}$ Chun-Cheng et al (32\%), ${ }^{5}$ and Peker et al (87.5\%). ${ }^{4}$ Although our control cohort was being imaged because of unexplained neurologic symptoms, these subjects probably reflect the incidence of vascular contact/compression of the vagus nerve in the general population.

During the past 20 years, our center has evaluated $>1500$ patients with trigeminal neuralgia and hemifacial spasm. MR imaging plays an important role in their surgical evaluation. Once the diagnosis is confirmed by history and physical examination, the presence of arterial compression of the appropriate nerve triggers a discussion of MVD with the patient. It also provides critical information for the surgeon to plan the procedure and anticipate the intraoperative findings. Absence of compression halts the consideration of MVD because there is nothing to decompress. The MR imaging documentation of neurovascular compression is therefore a necessary but not sufficient finding to justify MVD of the nerve. We believe the MR imaging investigation of patients with HELPS will be similar. The high incidence of asymptomatic vascular contact/compression of the vagus nerve $(\sim 50 \%)$ means that its presence cannot be used as a definitive test for this condition. The absence of a contact/compression, however, can be used to rule out the condition.

Limitations of our study include the small number of patients with HELPS; the simplicity of our rating scheme not distinguishing different degrees of compression, including possible dis- 
tortion or atrophy of the nerve; and the study being performed on a $1.5 \mathrm{~T}$ MR imaging platform, rather than at $3 \mathrm{~T}$ (which may have allowed better vessel-to-nerve characterization).

\section{CONCLUSIONS}

Clinicians should be aware that the presence of unilateral neurovascular contact or compression of the vagus nerve does not confirm the diagnosis of HELPS because $50 \%$ of the general population may have such a finding. An accurate clinical history with a unilateral beneficial response to botulinum toxin in the vocal fold and ipsilateral vascular contact/compression of the vagus nerve should prompt a discussion about MVD. The MR imaging finding of ipsilateral vascular compression of the vagus nerve is therefore a necessary but not sufficient finding for the diagnosis of this rare condition.

\section{REFERENCES}

1. Honey CR, Gooderham P, Morrison M, et al. Episodic hemilaryngopharyngeal spasm (HELPS) syndrome: case report of a surgically treatable novel neuropathy. J Neurosurg 2017;126:1653-56 CrossRef Medline

2. Honey CR, Morrison M, Heran MKS, et al. Hemi-laryngopharyngeal spasm (HELPS): a novel cause of inducible laryngeal obstruction with a surgical cure. J Neurosurg 2018 Jul 20:1-5. [Epub ahead of print] CrossRef Medline

3. Suzuki M, Yoshino N, Shimada M, et al. Trigeminal neuralgia: differences in magnetic resonance imaging characteristics of neurovascular compression between symptomatic and asymptomatic nerves. Oral Surg Oral Med Oral Pathol Oral Radiol 2015;119:113-18 CrossRef Medline

4. Peker S, Dinçer A, Necmettin Pamir M. Vascular compression of the trigeminal nerve is a frequent finding in asymptomatic individuals:
3-T MR imaging of 200 trigeminal nerves using 3D CISS sequences. Acta Neurochir (Wien) 2009;151:1081-88 CrossRef Medline

5. Chun-Cheng Q, Qing-Shi Z, Ji-Qing Z, et al. A single-blinded pilot study assessing neurovascular contact by using high-resolution $M R$ imaging in patients with trigeminal neuralgia. Eur J Radiol 2009;69: 459-63 CrossRef Medline

6. Leal PR, Hermier M, Froment JC, et al. Preoperative demonstration of the neurovascular compression characteristics with special emphasis on the degree of compression, using high-resolution magnetic resonance imaging: a prospective study, with comparison to surgical findings, in $\mathbf{1 0 0}$ consecutive patients who underwent microvascular decompression for trigeminal neuralgia. Acta Neurochir (Wien) 2010;152:817-25 CrossRef Medline

7. Zhou Q, Liu ZL, Qu CC, et al. Preoperative demonstration of neurovascular relationship in trigeminal neuralgia by using $3 \mathrm{D}$ FIESTA sequence. Magn Reson Imaging 2012;30:666-71 CrossRef Medline

8. Słoniewski P, Korejwo G, Zieliński P, et al. Measurements of the Obersteiner-Redlich zone of the vagus nerve and their possible clinical applications. Folia Morphol (Warsz) 1999;58:37-41 Medline

9. Guclu B, Sindou M, Meyronet D, et al. Cranial nerve vascular compression syndromes of the trigeminal, facial and vago-glossopharyngeal nerves: comparative anatomical study of the central myelin portion and transitional zone-correlations with incidences of corresponding hyperactive dysfunctional syndromes. Acta Neurochir (Wien) 2011;153:2365-75 CrossRef Medline

10. Sarrazin JL, Toulgoat F, Benoudiba F. The lower cranial nerves: IX, X, XI, XII. Diagn Interv Imaging 2013;94:1051-62 CrossRef Medline

11. Garcia M, Naraghi R, Zumbrunn T, et al. High-resolution 3D-constructive interference in steady-state MR imaging and 3D time-offlight MR angiography in neurovascular compression: a comparison between 3T and 1.5T. AJNR Am J Neuroradiol 2012;33:1251-56 CrossRef Medline

12. Antonini G, Di Pasquale A, Cruccu G, et al. Magnetic resonance imaging contribution for diagnosing symptomatic neurovascular contact in classical trigeminal neuralgia: a blinded case-control study and meta-analysis. Pain 2014;155:1464-71 CrossRef Medline 